\title{
Serum Neopterin Level Predicts HIV-Related Mortality but Not Progression to AIDS or Development of Neurological Disease in Gay Men and Parenteral Drug Users
}

Ned Sacktor, MD; Xinhua Liu, PhD; Matei Popescu, MD; Karen Marder, MD, MPH; Yaakov Stern, PhD; Richard Mayeux, MD, MSE

Objective: To investigate the ability of elevated serum neopterin levels to predict independently mortality, progression to acquired immunodeficiency syndrome, and development of neurological disease.

Design: Cross-sectional and longitudinal study of gay and/or bisexual men and parenteral drug users.

Setting and Patients: Patients included human immunodeficiency virus (HIV)-negative and -positive gay and/or bisexual men and parenteral drug-using men and women who volunteered for an outpatient study of the natural history of HIV infection.

Results: Serum neopterin levels were significantly elevated in HIV-positive patients (mean, $18.0 \mathrm{nmol} / \mathrm{L} ; \mathrm{SD}$, $19.2 \mathrm{nmol} / \mathrm{L})$, compared with those in HIV-negative patients (mean, $7.5 \mathrm{nmol} / \mathrm{L}$; SD, $5.5 \mathrm{nmol} / \mathrm{L})(P<.001)$. No differences in the serum neopterin levels could be detected between gay men and parenteral drug users. In HIVpositive patients, women had a higher serum neopterin level than did men $(P=.03)$. The elevated serum neopterin levels were associated with an advanced clinical stage of HIV infection. After adjusting for the CD4 lymphocyte count and other potential confounders, the serum neopterin level was a significant independent predictor of mortality. The elevated serum neopterin levels did not predict progression to acquired immunodeficiency syndrome or development of clinically significant neurological disease.

Conclusion: An elevated serum neopterin level predicts mortality, but it does not predict progression to acquired immunodeficiency syndrome or development of neurological disease among HIV-infected individuals.

(Arch Neurol. 1995;52:676-679)
From the Gertrude H. Sergievsky Center

(Drs Sacktor, Popescu, Marder, Stern, and Mayeux), the Human Immunodeficiency Virus Center for Clinical and Behavioral Studies, New York State Psychiatric Institute (Drs Sacktor, Liu, Marder, Stern, and Mayeux), and the Departments of Neurology (Drs Sacktor, Marder, Stern, and Mayeux) and Psychiatry (Drs Stem and Mayeux), Columbia University College of Physicians and Surgeons, New York. Dr Sacktor is presently with the Johns Hopkins Bayview Medical Center, Baltimore, Md.

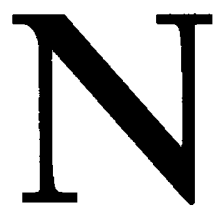

EOPTERIN, derived from guanosine triphosphate, is an intermediate product in the synthesis of tetrahydrobiopterin, which is an essential cofactor for tyrosine and tryptophan hydroxylation. ${ }^{1}$ Neopterin is produced by monocytes and macrophages during immune activation, and the level of serum neopterin is elevated in viral and bacterial infections, in cancer, and during allograft rejection. ${ }^{2}$ Macrophages and microglia are principal cell types that support productive human immunodeficiency virus (HIV) infection, ${ }^{3,4}$ and products of these cells, such as neopterin, may be an indicator of neurological disease. ${ }^{5}$

Elevated serum neopterin levels are potential prognostic markers in HIV infection for mortality ${ }^{1,6}$ and progression to acquired immunodeficiency syndrome (AIDS).$^{1,2,7-10}$ However, the ability of serum neopterin levels to predict the prognosis independent of potential confounders, such as the CD4 lymphocyte count, is in doubt. ${ }^{11}$ To our knowledge, the ability of serum neopterin levels to predict the development of HIV-related neurological disease has not been evaluated previously. This study assessed the value of the serum neopterin level as an independent prognostic indicator for mortality, the transition to AIDS, and the development of HIV-related neurological disease in both gay men and parenteral drug users.

\section{RESULTS}

Serum neopterin levels were measured in 140 gay and/or bisexual men and in 129 men and women who were drug users at baseline (Table 1). Elevated serum neopterin levels were significantly associated with the HIV serostatus (HIV-positive pa-

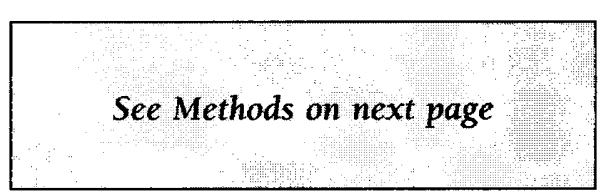




\section{METHODS}

Patients included HIV-negative and -positive gay and/or bisexual men and parenteral drug users of both sexes who volunteered for a study of the natural history of HIV infection. Patients were recruited via word of mouth at an infectious disease clinic and at a methadone maintenance clinic and via two announcements that were made in gay monthly newsletters and one announcement that was made in a newspaper. Inclusion and exclusion criteria and semiannual standardized medical, neurological, and neuropsychological evaluations have been described elsewhere. ${ }^{12-14}$

Blood tests included HIV serology that was performed by using an enzyme-linked immunosorbent assay and confirmed by Western blot, T-cell subset counts with a fluorescent cell sorter (EPIC-C, Coulter Corp, Hialeah, Fla), a p24 antigen assay with a standard kit (DuPont, Wilmington, Del), and an acid-labile interferon alfa titer (method available on request). The serum neopterin level was determined from plasma specimens that were frozen for storage at $-70^{\circ} \mathrm{C}$ by using a radioimmunoassay kit (Neopterin I RIA Kit, manufactured by IBL, Germany, for INCSTAR Corp, Stillwater, Minn).

In a cross-sectional study, we investigated the relationship between serum neopterin levels and clinical and laboratory characteristics that were assessed at each patient's first examination. In a longitudinal study, we analyzed the capacity of the baseline serum neopterin level to predict the following outcomes: mortality; progression to AIDS as defined by the 1987 criteria of the Centers for Disease Control and Prevention, Atlanta, $\mathrm{Ga}^{15}$; and development of neurological disease. All patients who died during the 31/2-year period of follow-up were included in the analysis. The date on which AIDS developed was assumed to have occurred at the midpoint between the prior evaluation and the first follow-up visit when AIDS was present, or at the midpoint between the prior evaluation and death.
A summary score to reflect the overall degree of neurological disability was calculated based on a modified version of the Kurtzke Disability Status Scale for Multiple Sclerosis (ie, Kurtzke score). ${ }^{14,16}$ Meaningful neurological disease was considered present if the Kurtzke score worsened from 0 to 2 (no or minimal disability) at baseline, to 3 (moderate disability although fully ambulatory) or greater during follow-up. Findings from the neuropsychological testing were defined as abnormal based on the neuropsychologists' clinical impression of the full battery of tests, as described previously. ${ }^{14}$

Wilcoxon's rank sum test was used to assess differences in serum neopterin levels between the two different subgroups, eg, HIV-positive and HIV-negative patients. To assess differences among the three different subgroups, the Kruskal-Wallis test was performed. The level of serum neopterin was dichotomized into groups with a high and low level of serum neopterin, by using the median in all HIVpositive patients. Comparisons of variables of interest in the two groups (those patients with high and low levels of serum neopterin) were performed either by using Wilcoxon's rank sum test for continuous variables with a skewed distribution ${ }^{17}$ or the $\chi^{2}$ test with Yates' Correction for Continuity for categorical variables. ${ }^{18}$ Variables that were shown to be significantly associated with the serum neopterin level at baseline or clinically significant variables (eg, age) were investigated further, by using logistic regression. ${ }^{19}$ Continuous variables were converted to binary forms with cutoff points that corresponded to the median of the HIVpositive population, except for the CD4 lymphocyte count, for which a cutoff value of $0.20 \times 10^{9} / \mathrm{L}(200 / \mu \mathrm{L})$ was chosen because of the AIDS-defining significance of this cutoff value. The risk ratios for death, AIDS, or neurological disability for the level of serum neopterin were calculated by using Cox proportional hazards models. ${ }^{20}$ The mortality risk ratio was adjusted for variables that were independent predictors of mortality and were significant in predicting high serum neopterin levels at baseline. tients [mean, $18.0 \mathrm{nmol} / \mathrm{L}$; SD, $19.2 \mathrm{nmol} / \mathrm{L}$ ] vs $\mathrm{HIV}$ negative patients [mean, $7.5 \mathrm{nmol} / \mathrm{L} ; \mathrm{SD}, 5.5 \mathrm{nmol} / \mathrm{L}]$; $P<.001$ ). Serum neopterin levels did not differ by risk group (gay men vs parenteral drug users) among HIVnegative or -positive patients. Serum neopterin levels did not differ by gender among HIV-negative patients. Among HIV-positive patients, women had a significantly higher serum neopterin level than did men (mean, 22.5 vs 17.5 $\mathrm{nmol} / \mathrm{L} ; P=.03$ ). After adjustment for gender, age, and risk group by using logistic regression, the odds ratio (OR) for HIV seropositivity associated with high serum neopterin levels (above the median in HIV-positive patients, $12.2 \mathrm{nmol} / \mathrm{L}$ ) was 9.6 (95\% confidence interval $[\mathrm{CI}], 4.5$ to 20.7). High serum neopterin levels were associated with advanced clinical stages (ie, AIDS or AIDS-related complex, compared with those in less symptomatic HIVpositive patients ${ }^{21}$; however, high serum neopterin levels were inversely related to a mean CD4 lymphocyte count and hemoglobin level (Table 2). After adjusting for the effect of the CD4 lymphocyte count and hemoglobin level, the OR for an advanced clinical stage of HIV infection (ie, AIDS or AIDS-related complex) associated with a high serum neopterin level was 2.2 (95\% CI, 1.1 to 4.5 )

Forty-six deaths (all related to HIV) occurred between the baseline visit and the end of the study among 176 HIV-positive patients. Thirty-six deaths occurred among the 89 patients with high serum neopterin levels at baseline, and 10 deaths occurred among the $87 \mathrm{pa}-$ tients with low serum neopterin levels. Cox proportional hazards models were used to assess the mortality risk that was associated with the serum neopterin level. The unadjusted mortality risk ratio that was associated with high serum neopterin levels was 5.5 (95\% CI, 2.6 to 11.9$)$. The CD4 lymphocyte count $\left(\leq 0.20 \times 10^{9} / \mathrm{L}\right)$, hemoglobin level $(\leq 134.5 \mathrm{~g} / \mathrm{L} \mathrm{g} / \mathrm{dL})$, interferon alfa titer $(>1: 4)$, and advanced clinical stage (AIDS or AIDSrelated complex) were all predictors of mortality and were also associated with high serum neopterin levels at baseline. After adjusting for these four variables, the CD4 lymphocyte count (risk ratio, 6.4; 95\% CI, 2.9 to 14.0 ; $P<.001$ ) and high serum neopterin levels (risk ratio, 2.4; $95 \% \mathrm{CI}, 1.1$ to $5.7 ; P=.04$ ) were still significant predictors of mortality. In other words, an HIV-positive pa- 


\begin{tabular}{|c|c|c|c|c|}
\hline \multirow[b]{2}{*}{ Subgroups } & \multirow[b]{2}{*}{$\mathbf{n}$} & \multicolumn{2}{|c|}{$\begin{array}{l}\text { Serum Neopterin } \\
\text { Level, nmol/L }\end{array}$} & \multirow[b]{2}{*}{$P$} \\
\hline & & Mean & SD & \\
\hline \multicolumn{5}{|l|}{ HIV status } \\
\hline All negatives & 93 & 7.5 & 5.5 & \\
\hline All positives & 176 & 18.0 & 19.2 & $<.001$ \\
\hline \multicolumn{5}{|l|}{ Risk group } \\
\hline HIV-/gay men & 43 & 8.0 & 6.1 & $\ldots$ \\
\hline HIV-/PDU men & 31 & 6.0 & 2.8 & $\ldots$ \\
\hline HIV-IPDU women & 19. & 8.4 & 6.4 & .211 \\
\hline \multicolumn{5}{|l|}{ Gender } \\
\hline HIV+/gay men & 97 & 19.2 & 22.8 & $\ldots$ \\
\hline HIV+/PDU men & 48 & 14.2 & 12.9 & $\cdots$ \\
\hline HIV+/PDU women & 31 & 22.1 & 24.0 & .046 \\
\hline
\end{tabular}

* HIV indicates human immunodeficiency virus; PDUs, parenteral drug users; minus sign, seronegative; PDU, parenteral drug-using; and plus sign, seropositive.

tient with a CD4 count of $0.20 \times 10^{9} / \mathrm{L}$ or less was six times more likely to die during the next $3 \frac{1 / 2}{2}$ years than a patient with a CD4 count of greater than $0.20 \times 10^{\%} / \mathrm{L}$, and a patient with a CD 4 count of $0.20 \times 10^{\circ} / \mathrm{L}$ or less and a high serum neopterin level was 15 times more likely to die than a patient with a CD4 count of greater than $0.20 \times 10^{9} / \mathrm{L}$ and a low serum neopterin level. After adjusting for the CD4 lymphocyte count and the serum neopterin level, hemoglobin and interferon alfa values and an advanced clinical stage were no longer predictors of mortality. There was no significant interaction of the serum neopterin level and CD4 lymphocyte count in predicting mortality $(P>.10)$.

Seventeen of 30 patients whose conditions progressed to AIDS during the study period had a baseline serum neopterin level greater than $12.2 \mathrm{nmol} / \mathrm{L}$, and 13 had baseline serum neopterin levels below this value. The risk ratio for progression to AIDS in patients with high serum neopterin levels when compared with that in persons with low serum neopterin levels at baseline was not significant (ie, risk ratio, $1.5 ; 95 \% \mathrm{CI}, 0.7$ to 3.1 ).

Twenty-seven of 75 patients experienced significant neurological disease in the group with high serum neopterin levels, compared with 24 of 78 patients in the group with low serum neopterin levels. The risk ratio for development of significant neurological disease in patients with high serum neopterin levels, compared with that for patients with low serum neopterin levels at baseline, was not significant (ie, risk ratio, $1.4 ; 95 \% \mathrm{CI}, 0.8$ to 2.5 ).

\section{COMMENI}

In this study, elevated serum neopterin levels were independently associated with an advanced clinical stage at baseline and were inversely related to a mean CD4 lymphocyte count and hemoglobin level. Serum neopterin levels were significantly higher in HIV-positive than in HIV-negative patients, but no difference in serum neopterin levels were found among gay men and parenteral
Table 2. Comparison of Demographic and Clinical Characteristics in HIV-Seropositive Gay Men and PDUs With High vs Low Serum Neopterin Levels at Baseline Visit*

\begin{tabular}{|c|c|c|c|}
\hline & \multicolumn{2}{|c|}{ Serum Neopterin Level, nmol/ } & \multirow[b]{2}{*}{$P$} \\
\hline & $>12.2(n=89)$ & $<12.2(n=87)$ & \\
\hline \multicolumn{4}{|l|}{ Age, y } \\
\hline Mean (SD) & $39.3(7.12)$ & $38.4(8.36)$ & .402 \\
\hline \multicolumn{4}{|l|}{ Sex, \% } \\
\hline$F$ & 20.2 & 14.9 & \\
\hline$M$ & 79.8 & 85.1 & .470 \\
\hline \multicolumn{4}{|l|}{ Risk group, \% } \\
\hline Gay men & 55.1 & 55.2 & \\
\hline PDUs & 44.9 & 44.8 & 1.000 \\
\hline \multicolumn{4}{|l|}{ Clinical stage, $\%$} \\
\hline AIDS & 8.0 & 3.7 & .333 \\
\hline AIDS or ARC & 72.7 & 43.2 & $<.001$ \\
\hline \multicolumn{4}{|l|}{ CD4 lymphocyte count } \\
\hline Mean (SD), $\times 10^{\circ} / \mathrm{L}$ & $0.29(0.23)$ & $0.44(0.22)$ & $<.001$ \\
\hline$\leq 0.20 \times 10^{9} / \mathrm{L}, \%$ & 42.5 & 14.1 & $<.001$ \\
\hline \multicolumn{4}{|l|}{ Hemoglobin level } \\
\hline Mean (SD), $g / L$ & $127(15.9)$ & $139(13.7)$ & $<.001$ \\
\hline$\leq 134.5 \mathrm{~g} / \mathrm{L}, \%$ & 65.9 & 33.3 & $<.001$ \\
\hline \multicolumn{4}{|l|}{ Interferon alfa titer } \\
\hline$(>1: 4), \%$ & 47.5 & 26.3 & .010 \\
\hline p24 antigenemia & & & \\
\hline$(\geq 9), \%$ & 21.3 & 13.0 & .246 \\
\hline \multicolumn{4}{|l|}{ Kurtzke score } \\
\hline$(\geq 3), \%$ & 16.5 & 11.1 & .439 \\
\hline \multicolumn{4}{|l|}{ Abnormal } \\
\hline $\begin{array}{l}\text { neuropsychological } \\
\text { testing results, \% }\end{array}$ & 40.2 & 29.6 & .202 \\
\hline
\end{tabular}

*HIV indicates human immunodeficiency virus; PDUS, parenteral drug users; AIDS, acquired immunodeficiency syndrome; and ARC, AIDS-related complex.

drug users. This finding fails to support the hypothesis that the level of serum neopterin, an indicator of immune activation, might be elevated in drug users as a result of continuous antigen exposure. Among HIVpositive patients, women had higher serum neopterin levels than did men. Further investigation to evaluate gender differences in elevated serum neopterin levels is warranted.

The results of this study confirm those of other studies, ${ }^{1,6}$ suggesting that elevated serum neopterin levels predict mortality. After adjusting for potential confounders (CD4 lymphocyte count, hemoglobin and interferon alfa levels, and advanced clinical stage), high serum neopterin levels remained a significant predictor of mortality.

However, high serum neopterin levels did not predict the development of neurological disease. In contrast to the findings in other studies, ${ }^{1,2,7-10}$ we found that the serum neopterin level was not a predictor of progression to AIDS. The small number of patients in whom AIDS developed in our study could account for the discrepancy between our results and those of previous studies. In addition, patients who did not return for follow-up visits were interviewed by telephone to obtain further data on mortality only.

The present analysis was based on data from a prevalence sample of HIV-infected patients who were followed up longitudinally and whose duration of infec- 
tion, an important prognostic factor, ${ }^{7,22,23}$ was not known. It is possible that the predictive value of serum neopterin levels changes during the course of HIV infection. ${ }^{\text {? }}$ This could possibly explain the discrepancy between our results and those of previous studies concerning the independent predictive value of the serum neopterin level.

An elevated serum neopterin level is associated with an advanced clinical stage (AIDS or AIDS-related complex) at baseline, and it predicts mortality after adjusting for the CD4 lymphocyte count and other potential confounders. In our study, elevated serum neopterin levels did not predict progression to AIDS or development of neurological disease.

Accepted for publication August 25, 1994.

This study was supported in part by project 817 5332C-7001 from the Aaron Diamond Foundation, New York, NY, by center grant P50-1-MH43520 from the National Institute of Mental Health/National Institute on Drug Abuse, and by grant T32-MH19139-05 from the National Institute for Mental Health to the Human Immunodeficiency Virus Center for Clinical and Behavioral Studies, Bethesda, Md.

Reprint requests to Gertrude H. Sergievsky Center, Columbia University College of Physicians and Surgeons, $630 \mathrm{~W}$ 168th St, New York, NY 10032 (Dr Mayeux).

\section{REFERENCES}

1. Fuchs D, Hausen A, Reibnegger $G$, et al. Neopterin as a marker for activated cell-mediated immunity: application in HIV infection. Immunol Today. 1988;9: 150-154.

2. Ellaurie $M$, Calvelli $T$, Rubinstein A. Neopterin concentrations in pediatric human immunodeficiency virus infection as predictor of disease activity. Pediatr Infect Dis J. 1992;112:286-289.

3. Koenig S, Gendelman HE, Orenstein JM, et al. Detection of AIDS virus in macrophages in brain tissue from AIDS patients with encephalopathy. Science. 1986;233:1089-1093.

4. Wiley CA, Schrier RD, Nelson JA, et al. Cellular localization of human immunodeficiency virus infection within the brains of acquired immune deficiency patients. Proc Natl Acad Sci U S A. 1986;83:7089-7093.
5. Griffin DE, MCArthur JC, Cornblath DR. Neopterin and interferon-gamma in serum and cerebrospinal fluid of patients with HIV-associated neurologic disease. Neurology. 1991;41:69-74.

6. Jacobson MA, Bacchetti $P$, Kolokathis $A$, et al. Surrogate markers for survival in patients with AIDS and AIDS related complex treated with zidovudine. BMJ. 1991;302:73-78.

7. Kramer A, Biggar RJ, Hampl H, et al. Immunologic markers of progression to acquired immunodeficiency syndrome are time dependent and illness specific. Am J Epidemiol. 1992:136:71-80.

8. Munoz A, Vlahov D, Solomon $L$, et al. Prognostic indicators for development of AIDS among intravenous drug users. J Acquir immune Defic Syndr. 1992; 5:694-700.

9. Melmed RN, Taylor JMG, Detels R, Bozorgmehri M, Fahey JL. Serum neopterin changes in HIV-infected subjects: indicator of significant pathology, CD4 T-cell change and development of AIDS. J Acquir Immune Defic Syndr. 1989;2:70-76.

10. Osmond DH, Shiboski S, Bacchetti $P$, Winger EE, Moss AR. Immune activation markers and AIDS prognosis. AIDS. 1991;5:505-511.

11. Diagnostic and therapeutic technology assessment: surrogate markers of progressive HIV disease. JAMA. 1992;267:2948-2952.

12. Gorman JM, Kertzner $R$, Todak $G$, et al. Multidisciplinary baseline assessment of homosexual men with and without human immunodeficiency virus infection, I: overview of study design. Arch Gen Psychiatry. 1991;48:120-123.

13. El-Sadr W, Goetz RR, Sorrell S, et al. Clinical and laboratory correlates of HIV infection in a cohort of intravenous drug users from New York City. Arch intern Med. 1992;152:1653-1659.

14. Stern $Y$, Marder $K$, Bell $K$, et al. Multidisciplinary baseline assessment of homosexual men with and without human immunodeficiency virus infection, III: neurologic and neuropsychological findings. Arch Gen Psychiatry. 1991;48: 131-138.

15. Centers for Disease Control. Classification system for human T-lymphotropic virus type III/lymphadenopathy-associated virus infections. MMWR Morb Mortal Wkly Rep. 1986;35:334-339.

16. Kurtzke J. Rating neurological impairment in muitiple sclerosis: an expanded disability scale (EDSS). Neurology. 1983;33:1444-1452.

17. Daniel WW. Biostatistics: A Foundation for Analysis in the Health Sciences. 5th ed. New York, NY: John Wiley \& Sons Inc; 1987.

18. Fleiss JL. Statistical Methods for Rates and Proportions. 2nd ed. New York, NY: John Wiley \& Sons Inc; 1981

19. Werner J. Medizinische Statistik. Baltimore, Md: Urban \& Schwarzenberg; 1984.

20. Cox DR. Regression models and life-tables. J R Stat Soc B. 1972;34:187-220.

21. Gorman J, Kertzner R, Spitzer R, Mayeux R. Development and characteristics of a medical staging system for HIV infection. Int $J$ Methods Psychiatr Res. 1992;2:117-124.

22. Krown SE, Niedzwiecki D, Bhalla RB, Flomenberg N, Chapman D. Relationship and prognostic value of endogenous interferon-alpha, beta 2-microglobin, and neopterin serum levels in patients with Kaposi sarcoma and AIDS. $J$ Acquir Immune Defic Syndr. 1991;4:871-880.

23. Moss AR, Bacchetti P. Natural history of HIV infection. AIDS. 1989;3:55-61.

\section{Announcement}

Free Patient Record Forms Available

Patient record forms are available free of charge to ARCHIVES readers by calling or writing FORMEDIC, 12D Worlds Fair Dr, Somerset, NJ 08873-9863, telephone (908) 469-7031. 